\title{
¿QUÉ PSICOTERAPIA?, ¿PARA QUIÉN? ALGUNOS AVANCES EN UN LARGO CAMINO
}

Ricardo Bernardi

Médico psiquiatra, magíster en Psicoanálisis y doctor en Psicología, UBA Miembro de Honor de APU Correo electrónico: ric.e.bernardi@gmail.com ORCID: 0000-0002-1117-5821 


\section{Sobre el texto y su autor}

El texto de la presente entrega de Relecturas fue escrito para una actividad preparatoria del $4 .{ }^{\text {to }}$ Congreso de AUDEPP, Desafios a la psicoterapia (mayo de 2001) y publicado en la Revista de Psicoterapia Psicoanalítica, tomo VI, número 2 (junio de 2002). En ese entonces, cuando la palabra globalización todavía parecía novedosa, AUDEPP se interrogaba sobre una clínica psicoanalítica interpelada por el desarrollo científico y tecnológico y su repercusión en la conformación de la subjetividad. También se interesaba por indagar sobre los nuevos campos de trabajo e investigación que se abrían y demandaban un diálogo con otras disciplinas. Asimismo, reclamaba la necesidad de incorporar la atención en salud mental a las coberturas asistenciales. En ese contexto de cambios, el Dr. Bernardi asumía, tal vez sin proponérselo, una suerte de liderazgo intelectual en el ámbito psicoanalítico local al insistir sobre la necesidad de dar cabida a la investigación sistemática dentro de la disciplina y de revalorizar la importancia del diagnóstico psicodinámico. En la adenda que el autor ha tenido la gentileza de redactar para enmarcar esta reedición del artículo original, el lector podrá seguir algunos frutos concretos de aquel empeño.

Ricardo Bernardi es médico psiquiatra, magíster en Psicoanálisis y doctor en Psicología de la Universidad de Buenos Aires. Es profesor emérito de la Facultad de Medicina de la Universidad de la República, miembro de la Academia Nacional de Medicina y miembro de honor de la Asociación Psicoanalítica del Uruguay y de la Sociedad de Psiquiatría del Uruguay. Es integrante del Grupo Asesor Científico Honorario de la Presidencia de la República, investigador grado 2 de la Agencia Nacional de Investigación e Innovación, así como vicepresidente del Comité de Investigación y consultor del Comité de Observación Clínica de la Asociación Psicoanalítica Internacional.

Entre sus muchas publicaciones cabe destacar, por su vinculación con este artículo, La formulación psicodinámica de caso: su valor para la práctica clínica (2016), libro cuya edición coordinó y del cual es coautor, junto a B. Varela, D. Miller, R. Zytner, L. de Souza y R. Oyenard. 
Las exposiciones que acabamos de oír nos ofrecieron una perspectiva sobre dos aspectos complementarios útiles para pensar el papel de las psicoterapias hoy en nuestro medio. Hemos visto cómo se construyó en la clínica psiquiátrica un espacio de intercambio entre distintas corrientes de psicoterapia, espacio que a su vez facilita la investigación en este campo. También escuchamos un panorama muy claro de lo que, para utilizar una expresión gráfica, constituye el «estado del arte» en cuanto a la integración de la psicoterapia y de la psicofarmacología, remarcando puntos de gran interés clínico.

Me gustaría centrarme, en el tiempo que dispongo, en algunos puntos problemáticos en torno a la psicoterapia en general y al psicoanálisis en particular, y a la forma en que la investigación sistemática puede ayudar a responder problemas de la práctica clínica. Me parece que esta es una forma de contribuir a la invitación que se me ha hecho para preparar el congreso de AUDEPP, invitación que mucho agradezco.

Para ir directamente al centro de la cuestión: ¿cuál recurso terapéutico usar para qué paciente? La respuesta parece obvia: el tratamiento que resulte más adecuado en cada caso. Sin embargo, las preguntas que surgen a continuación ya no son tan obvias. ¿En qué nos basamos para decir que es el mejor tratamiento? ¿Dónde encontrar la mejor evidencia disponible sobre la eficacia de los distintos tratamientos? Estas cuestiones nos llevan a preguntarnos en base a qué criterios organizamos la atención a las personas que solicitan nuestra ayuda, cómo tomamos en cuenta los distintos recursos existentes y, en forma más específica, qué es lo que los pacientes pueden esperar de la psicoterapia. 
Si tomamos en cuenta el título de esta jornada, que es «Los problemas de hoy día», vemos que existen problemas abiertos a discusión tanto con relación a lo que las personas esperan de la psicoterapia como con relación a sus resultados. La psicoterapia se enfrenta a cambios en la concepción misma de la subjetividad. El mundo llamado posmoderno no es el mundo de la modernidad en cuanto a la manera como el hombre siente y maneja su propia interioridad. La noción misma de lo interno o privado ha cambiado y el hombre o la mujer no siempre sienten que su propio mundo interior es un lugar que invita a ser explorado. La distinción interno-externo y privado-público es manejada con criterios culturales muy distintos a los que existían en el momento en que nació el psicoanálisis. Las personas son invitadas a verse más a través de la acción y del reflejo en los demás, que a través de la búsqueda interior. Esto puede desembocar tanto en una preocupación auténtica y positiva por las relaciones sociales, como en el intento de guiarse por la imagen pública más que por los aspectos verdaderos del self. El primer problema, por tanto, es el de la relación con la propia interioridad. En consecuencia, es necesario preguntarse por la demanda del paciente y poder trabajar con él en qué medida esa demanda resulta terapéutica o antiterapéutica, si apunta al crecimiento personal o no.

A su vez, el campo del psicoanálisis y la psicoterapia han cambiado. Tenemos diferentes teorías psicoanalíticas, diferentes técnicas psicoterapéuticas y múltiples recursos disponibles: farmacológicos, sociales, etcétera. Entonces inevitablemente se plantea la pregunta: ¿cómo podemos saber y demostrar cuál tratamiento es el mejor para cada quien?

Otra cosa que cambió es que ahora está claro que los colegas -y también los pacientes - tienen derecho a preguntarnos en qué nos basamos para aconsejar un tratamiento en vez de otro. Antes bastaba que uno practicara determinada técnica psicoterapéutica para que la recomendara prácticamente a todo el mundo. Ahora nadie puede cuestionar que 
un paciente o un colega nos pueda preguntar: ¿y de dónde saca que para tal trastorno lo mejor es este tratamiento? O sea, ¿de dónde proviene su evidencia?

Este tema es más general. Existe un movimiento dentro de la medicina actual, llamado Medicina Basada en Pruebas o en la Evidencia, según cómo se traduzca (Evidence-based Medicine), que busca responder a este tipo de preguntas, o sea, el de cuál es la mejor evidencia disponible para recomendar un tratamiento frente a otros posibles. Este movimiento surge de distintas fuentes. Por un lado, está la preocupación por respetar los derechos de los usuarios. También la intención de racionalizar los costos de la salud. Por otro lado, el avance de la epidemiología clínica permitió comparar diferentes tratamientos estudiados en múltiples investigaciones y, a través de técnicas estadísticas, combinar los datos de esas distintas investigaciones para saber cada vez con mayor precisión qué efecto tiene cada tratamiento. Notemos que esto se une a la idea de una enseñanza basada en problemas y no en temas, lo cual lleva a jerarquizar los conocimientos que son necesarios para la toma de decisiones en situaciones relacionadas con la práctica diaria.

La pregunta siguiente es: ¿en base a qué tipo de evidencia o pruebas estamos intentando resolver los problemas y tomar las decisiones? El movimiento hacia una práctica basada en pruebas incluye las psicoterapias y se expresa en la pregunta acerca de cuáles son los tratamientos psicoterapéuticos sustentados empíricamente.

Primero se habló de tratamientos validados empíricamente, pero el término validados es demasiado exigente, por lo cual se habló en forma menos exigente de tratamientos sustentados empíricamente (empirically supported), es decir, con base empírica y apoyados por estudios científicos. Esto no quiere decir que se deba partir de conclusiones inmodificables, sino todo lo contrario: de resultados continuamente revisados en base a los nuevos estudios que se van realizando. 
La pregunta relevante la resumen muy bien Roth y Fonagy (1996) en un libro titulado precisamente ¿Qué funciona para quién? (What works for whom?), en el cual hacen una revisión crítica de las investigaciones disponibles sobre los distintos tratamientos psicoterapéuticos. Este es uno de los trabajos más claros que intenta responder esta pregunta.

¿Dónde colocar en todo esto a la evidencia que se va generando en la práctica clínica, esto es, la opinión que se forman los clínicos sobre los resultados de los tratamientos que realizan? Sin duda, este tipo de opiniones debe ser tenido en cuenta, pero con clara conciencia de sus limitaciones. Los juicios clínicos individuales son insuficientes: los clínicos por lo general no podemos investigar sistemáticamente los efectos de los tratamientos que realizamos, no hacemos estudios de seguimiento y no tenemos forma de decidir cuando hay dos opiniones distintas, salvo que recurramos a criterios de autoridad. Necesitamos, por tanto, complementar la investigación científica sistemática, que compara los distintos tratamientos en forma rigurosa, buscando controlar los factores que pueden introducir sesgos y llevar a falsas conclusiones. Idealmente, ambos criterios deberían coincidir, pero no siempre ocurre así ni en el campo de la psicoterapia ni en los otros campos de la salud.

El problema es, entonces, el de cómo articular la experiencia clínica individual con los estudios sistemáticos para lograr la mayor seguridad de que estamos haciendo lo mejor para el paciente.

Hay algo que debe destacarse: no siempre la formación habitual en psicoterapia brinda los criterios necesarios para esta evaluación. Es frecuente que, en psicoterapia analítica o de otro tipo, el criterio de validez de las intervenciones del terapeuta lo constituya un determinado modelo ideal de cómo debería ser el proceso terapéutico. Un analista lacaniano se sentirá muy inclinado a tomar las obras de Lacan como guía para sus intervenciones, uno winnicottiano las de Winnicott, uno cognitivo-conductual las de Beck, y así sucesivamente. Puede haber mezclas más o menos 
coherentes entre estos distintos modelos, pero todos ellos inducen al terapeuta a pensar que lo mejor que puede hacer por el paciente es seguir el modelo correctamente. Sin duda, es importante que un psicoterapeuta tenga un buen conocimiento de estos modelos teóricos y técnicos $-\mathrm{y}$ si puede conocer no solo los de su técnica, sino también los de otras técnicas, mejor-, pero este conocimiento de los modelos ideales no alcanza para estar seguro de los resultados. La pregunta entonces es: ¿cómo podemos comprobar que lo que hacemos le sirve al paciente?

El punto de partida, como dije, surge de la experiencia clínica individual o de aquellos a quienes reconocemos como nuestros supervisores o colegas. La frase tan común «Mira, a mí un caso similar me marchó muy bien encarándolo de tal o cual manera...» sigue siendo el punto de partida. Este es el nivel básico. Pero a veces la evidencia científica nos hace poner señales de advertencia o luces amarillas o incluso rojas en prácticas en las que tendemos a confiar en base al conocimiento clínico.

En realidad, hay muchas razones para limitar la validez de los juicios clínicos individuales. La casuística individual, o sea, el decir «En mi experiencia las cosas son así, de acuerdo a los pacientes que yo vi», no toma en cuenta que esos casos que uno vio pueden no ser en absoluto representativos del conjunto de los pacientes. La ilusión de los clínicos de la que habla a menudo el analista chileno Juan Pablo Jiménez tiene que ver con esta convicción de que lo que vimos en un caso vale para todos. O que nuestros criterios son los únicos que valen para encarar un problema. Déjenme poner un ejemplo, relacionado con el transexualismo.

Hace algunos años, comenzaron a llegar al Departamento de Psicología Médica pedidos de estudio para cirugía de reasignación de sexo en casos de transexualismo. O sea, pacientes con esta patología específica (que no debe ser confundida con la perversión o el delirio) que solicitaban un cambio de los caracteres sexuales por medio de la cirugía. Me sorprendió que mientras los estudios estadísticos realizados en otros medios mostraban 
resultados favorables a estos tratamientos quirúrgicos, en nuestro medio existían muchas opiniones contrarias. En estos casos corresponde preguntarse de dónde proviene la evidencia a favor o en contra. Encontré que los estudios publicados en otros medios estaban, por lo general, bien diseñados y controlados (a veces no), y el análisis del conjunto de ellos permitía sacar una conclusión favorable a la cirugía cuando se cumplían determinados requisitos diagnósticos y de psicoterapia previa. ¿De dónde provenían las opiniones contrarias? No encontré ninguna que estuviera sostenida por estudios similares; en realidad, se basaban en suposiciones derivadas de conocimientos psiquiátricos o psicoanalíticos o en observaciones parciales y no sistemáticas.

Analizando más a fondo el problema, era evidente que de las mismas teorías psicopatológicas podían derivarse distintas hipótesis alternativas, que implicaban conductas terapéuticas muy distintas. Quiero decir que muchas veces no prestamos atención al hecho de que de una misma teoría psicopatológica pueden derivarse distintas conclusiones, y que la única forma de saber cuál es la verdadera es la observación. En este caso, estudiamos a varios transexuales durante tratamientos psicoterapéuticos antes y después de la cirugía de reasignación de sexo y, aunque se trataba de casos con configuraciones psicológicas muy diferentes, en todos ellos pudo apreciarse una notoria mejoría de la calidad de vida. ¿Podemos decir que estas pocas observaciones permiten sacar conclusiones? Sí, dentro de los límites del inductivismo enumerativo. ¿Cómo podemos mejorar esta evidencia?

Tuve ocasión de comentar estos resultados con un psicoanalista de Hamburgo, Pfäfflin, autor de trabajos y libros sobre el tema. Cuando le pregunté cuántos casos tenía estudiados me comentó que más de quinientos, con resultados similares a los nuestros, que en aquel momento eran cuatro (ahora tenemos alguno más). ¿Valen quinientos más que cuatro? No, en cuanto a lo que podemos decir de esos cuatro, pero sí en cuanto 
al valor de la generalización. Si quisiéramos fortalecer aún más nuestras conclusiones, deberíamos pasar a un estudio clínico controlado y configurar un grupo sin tratamiento para compararlo con el tratado. Pero allí nos encontraríamos con problemas éticos y metodológicos similares a los que encuentran los estudios de resultados en psicoanálisis, porque no es posible dejar a personas sin tratamiento por años si no hay tratamiento alternativo comparable. Es posible, sin duda, ensayar nuevos diseños, pero lo que me interesa aquí es destacar la importancia de basarnos en datos controlados y comparables con los de otros medios.

Otro problema que presentan las observaciones clínicas es que los métodos de registro del material clínico son demasiado influenciables por variables que dependen del terapeuta y que escapan a la detección. Todos sabemos que el observador y el acto mismo de la observación influyen en lo observado. Por esa razón, si bien no podemos hacer desaparecer esta influencia, podemos diseñar metodologías que nos permiten conocerla y controlarla lo más posible. Hace más de treinta años, autores rioplatenses como Bleger o Liberman nos prevenían sobre estos problemas. David Liberman, en Buenos Aires, comenzó a apoyarse en el registro en audio de las sesiones para realizar el microanálisis del proceso analítico. Tanto él como José Bleger propusieron indicadores psicoanalíticos del avance del proceso analítico. Liberman buscaba el cambio a través de indicadores lingüísticos o semióticos, Bleger estudiaba los cambios en los grados de libertad del paciente en las conductas transferenciales. Estos son estudios pioneros desarrollados en el Río de la Plata en las décadas del sesenta y del setenta que, lamentablemente, fueron discontinuados porque nuestros países están muchas veces más inclinados a buscar lo novedoso que viene de afuera que a desarrollar sus propias ideas. Pero estaban indicando un camino que fue el que siguió la investigación en psicoterapia en otros medios.

Sin duda, hoy en día tenemos otros medios técnicos que pueden ser utilizados. En nuestro país, Silvia Gril, con el apoyo de la Universidad de 
Ulm y del Departamento de Psicología Médica, ha estudiado los cambios en los procesos de abstracción y emoción en el lenguaje del paciente, usando a esos efectos programas computacionales. Junto con Marina Altmann ha investigado las intervenciones breves madre-bebe, comparando el lenguaje de la madre, analizado con el método que recién mencioné, con los cambios en las conductas de apego con el bebe, estudiadas por medio de una escala aplicada a las videograbaciones de las entrevistas. Han cambiado sin duda los medios técnicos, pero el espíritu es el mismo que guiaba a Bleger o a Liberman.

Hasta aquí me referí a estudios del proceso terapéutico o de la relación proceso-resultados. Pero en el estudio de los resultados de la psicoterapia o del psicoanálisis ha ocurrido también una revolución en la última década. Por un lado, se dispone de numerosos instrumentos para evaluar el cambio de los pacientes, no solo en sus aspectos sintomáticos o globales (como el scL-90 o las escalas de funcionamiento en diferentes áreas), sino también en aspectos más específicos, como, por ejemplo, las escalas de narcisismo, de coherencia del self, de mecanismos de defensa, etcétera. Los resultados de estos instrumentos se han cotejado con las evaluaciones que el paciente mismo, el terapeuta y las terceras partes hacen de los cambios ocurridos. En una palabra, se sabe más de cómo evaluar los cambios. Pero a su vez se dispone de instrumentos estadísticos, como el metaanálisis, que permiten combinar estudios procedentes de distintas fuentes y obtener medidas de resumen que hacen posible llegar a conclusiones que toman en cuenta el conjunto de la información disponible en un momento dado. Como dije antes, estos son procedimientos que están en la base de la corriente actual de Medicina Basada en la Evidencia y que abarcan también el campo de la psicoterapia.

¿Adónde hemos llegado por este camino? Por un lado, tenemos los estudios de eficacia, que nos permiten comparar dos tratamientos realizados en forma ideal. Para decirlo gráficamente, el tribunal supremo en la 
comparación de tratamientos médicos está constituido por el metaanálisis de los ensayos clínicos controlados o randomizados, en los cuales dos tratamientos son comparados a ciegas en pacientes con idénticos trastornos. La suma de distintas investigaciones de este tipo permite evaluar la eficacia de estos tratamientos. Pero hay un problema: se ha comprobado que en la realidad de todos los días los tratamientos no se aplican en las condiciones ideales que rigen en los ensayos clínicos controlados (pacientes con los mismos trastornos, que cumplen el tratamiento fielmente, que son atendidos de igual manera, terapeutas con igual competencia y técnicas, etcétera). En la realidad los pacientes tienen alto grado de comorbilidad, no adhieren al tratamiento, el nivel y la calificación técnica de la asistencia varía, etcétera. Entonces, se hacen necesarios estudios de efectividad que investiguen los tratamientos en las condiciones reales o ecológicas en las que tienen lugar. Y esto se completa con los estudios de eficiencia, que examinan los resultados de los tratamientos en función del beneficio que brindan en proporción al esfuerzo que requieren (estudios de costo-beneficio, costo-utilidad, etcétera).

Todo esto va configurando un campo con cuyos procedimientos tanto la psicoterapia como los tratamientos farmacológicos deben aprender a moverse si quieren correr en esta carrera. Esto no es simplemente una conspiración de algunos administradores de salud que quieren bajar los costos complicando a los que honestamente nos ganamos la vida haciendo tratamientos psicoterapéuticos o farmacológicos. Por supuesto, como todo conocimiento, puede prestarse para manipulación y sin duda existen intereses de todo tipo en juego: económicos, corporativos, luchas de prestigio y poder, etcétera. Pero, primeramente, estos recursos científicos deben estar, antes que nada, a beneficio de los usuarios, porque la población necesita una garantía de que está recibiendo el mejor tratamiento disponible. Por eso, el núcleo al que hay que apuntar es que esta metodología sirva para orientar a técnicos y a la población sobre 
cuáles son los mejores tratamientos para sus problemas. Y debe servir para alertarnos de que a veces nuestras decisiones terapéuticas no están suficientemente fundamentadas.

Pongamos algún ejemplo sobre el problema de la mejor evidencia disponible. Si yo les digo: «Piensen en el mejor tratamiento para un paciente deprimido», es muy probable que muchos de ustedes piensen en psicoterapia asociada con farmacoterapia. Si yo les digo: «Se comprobó que con uno solo de los dos tratamientos alcanza», es muy probable, si la depresión es severa, que ustedes piensen en la farmacoterapia. Esa sería también mi reacción espontánea. Pero no debemos confiar en que lo que parece de sentido común siempre coincida con la mejor evidencia científica de la que disponemos. Más aun, es posible que muchos de nosotros pensemos que los estudios más rigurosos van siempre a favorecer a los psicofármacos y no a la psicoterapia. Hay algunas razones para que esto sea así: la metodología que requieren los estudios sobre resultados de la psicoterapia es más compleja y el financiamiento es más difícil de lograr que para hacer estudios sobre psicofármacos. Digamos al pasar que, como hay más estudios sobre psicofármacos que sobre psicoterapia, esto llevó a que se confundiera la ausencia de evidencia con la evidencia de la ausencia (de efectos terapéuticos de la psicoterapia).

En el caso que les dije es seguro que todos pensaríamos que no hay evidencia disponible a favor de utilizar la psicoterapia como único tratamiento en el caso de la depresión severa. Y nos equivocaríamos.

Es cierto que los estudios clásicos, tomados generalmente como referencia en los protocolos o guías para los tratamientos, como el realizado por el Instituto Nacional de Salud Mental de Estados Unidos (NIMH), a cargo de Elkin et al. (1989), sostienen que los psicofármacos superan en eficacia y rapidez a la psicoterapia en las depresiones agudas y severas. No así en las depresiones leves, donde los resultados se equiparan 
o donde son superiores los de ciertas formas de psicoterapia, y mucho menos en los duelos donde los psicofármacos no están indicados.

Pero, si uno empieza a revisar la bibliografía reciente, encuentra que incluso estas verdades que parecían incontrovertibles están cuestionadas. Por ejemplo, DeRubeis et al. (1999) sometieron los estudios manejados por Elkin et al. (1989) a estudios de metaanálisis y megaanálisis, y de estos surgió que los resultados de la psicoterapia eran comparables a la farmacoterapia en los casos de depresión aguda severa ambulatoria. Sin duda, este estudio aislado no alcanza para zanjar la cuestión y, de hecho, surgieron nuevos trabajos que replantearon el tema en función de los nuevos antidepresivos, etcétera. También desde el lado de las psicoterapias existen estudios que sugieren que las conclusiones del trabajo anterior, realizado para la psicoterapia cognitivo-conductual, debería replantearse para otras psicoterapias, como la dinámica o la interpersonal (Roth y Fonagy, 1996). Pero lo que quiero decir es que tenemos que cuidar de tomar nuestro buen sentido clínico como dogma.

Tampoco la receta ecléctica, o sea, la de salir de la duda recomendando sumar psicoterapia y farmacoterapia es del todo satisfactoria, porque se ha cuestionado que los dos tratamientos juntos sean siempre mejores que uno solo (Howard, comunicación personal). Entonces, no solo los administradores de salud van a poner el grito en el cielo porque duplicamos costos, sino que el paciente puede tener que someterse a tratamientos que no precisa y que pueden tener efectos secundarios indeseables (pensemos, por ejemplo, en las dificultades sexuales que pueden provocar los ISRS).

¿Adónde quiero llegar? Primero, a que tenemos que distinguir la práctica que realizamos porque es la más aceptada en determinado medio, de lo que es la mejor evidencia disponible. No estoy proponiendo que de buenas a primeras modifiquemos nuestro enfoque terapéutico de la depresión cada vez que se publique un nuevo trabajo, pero sí que pongamos allí una luz amarilla para indicar que hace falta estar más atentos a nuevos resultados. 
Volvamos ahora a la pregunta de Roth y Fonagy (1996), formulada en realidad por un investigador en psicoterapia, Paul, unos cuantos años antes: ¿cuál tratamiento, realizado por quién, es más efectivo para esta persona, que tiene cuáles problemas específicos y que se encuentra en qué circunstancias? Estas son las variables relevantes cuando uno tiene que decir qué tratamiento para quién.

¿Tenemos conocimientos firmes para responder a estas preguntas en el caso de la psicoterapia? Nuestra impresión clínica es que nadie puede dudar del efecto beneficioso de la psicoterapia. Sin embargo, en 1952, Eysenck, revisando los datos de diversos estudios, sostuvo que las psicoterapias mejoraban a los pacientes en la misma proporción que la remisión espontánea. O sea que los pacientes, cuando mejoraban, lo hacían en función del tiempo que pasaba y no por el tratamiento, porque muchos trastornos mentales eran fluctuantes y mejoraban solos. Hoy en día, estos resultados de Eysenck no son más sostenibles. Los mismos datos fueron reestudiados por Sperry et al. (1996) y por muchos otros, y se mostró que en un análisis más fino las psicoterapias realmente mejoraban a un mayor porcentaje de personas en menor tiempo que la remisión espontánea.

Aquí también fue necesario poner una luz amarilla para luego pasar a la verde cuando llegaron nuevos estudios: la efectividad de la psicoterapia está hoy fuera de duda. Pero la siguiente pregunta fue: ¿son igualmente eficaces todas las psicoterapias? Frente a esta pregunta hubo una serie de estudios que compararon los resultados de diferentes investigaciones. Resumiendo: no hay aún posibilidad contundente — con los instrumentos disponibles - de discriminar adecuadamente dentro del grupo de las principales psicoterapias.

Luborsky, para transmitir esta conclusión, usó una frase de Alicia en el país de las maravillas que se hizo famosa. En el libro de L. Carroll, el pájaro Dodo tiene que dar el veredicto en una carrera y dice: «Todos ganaron, 
todos merecen el premio» y este veredicto quedó como el mejor resumen del estado actual de la cuestión. Pero esta conclusión es insatisfactoria y no responde muchas preguntas: ¿son iguales en qué? y ¿de cuales psicoterapias estamos hablando? Fíjense que por lo menos los manuales registran 400, y algunos el doble, de técnicas psicoterapéuticas, de las cuales se estudiaron seriamente solo unas pocas. Y ahí empieza una serie de problemas a los que me gustaría referirme más en detalle. Hubo un informe realizado en Alemania por Meyer, que plantea que hay grandes familias de psicoterapia, lo que llama orientaciones básicas. Cree que, para incluir una psicoterapia como categoría, esta debe ofrecer una teoría general del campo de la salud y la enfermedad, tener suficiente amplitud para abarcar el conjunto de los fenómenos psicopatológicos y a la vez suficiente especificidad para señalar planteos diferenciales para diferentes situaciones clínicas, y quienes la practican deben tener una formación suficientemente acreditada y que sea específica para esa técnica. Con esos requisitos el número se reduce drásticamente, y quedan unas pocas familias u orientaciones básicas.

Los estadounidenses tomaron un camino distinto. Lo que piden son tratamientos manualizados, o sea, tratamientos estandarizados, guiados por pautas que figuran en manuales y que, por lo tanto, pueden reproducirse en forma igual, porque de este modo se puede saber que dos pacientes realmente están recibiendo el mismo tratamiento. Pero vamos a ver los problemas que trae esta última solución. ¿Es posible definir las psicoterapias de acuerdo a procedimientos estandarizados?

Vamos por partes. Sin duda hace falta algún tipo de manual interno propio de cada psicoterapeuta. Todos lo tenemos de alguna manera. Por otra parte, no se puede seriamente defender que no hace falta formación específica para realizar una técnica psicoterapéutica o que alcanza con la formación de grado en medicina, psiquiatría o psicología. Este es un punto en el que en nuestro medio nos debemos un debate franco. 
Pero, aunque admitamos que hace falta una formación básica, el tipo de manual interno al que se apunta es muy distinto según la psicoterapia de que se trate. Algunas técnicas, por ejemplo, la tradición más psicoanalítica y la psicoterapia centrada en el cliente o rogeriana, ponen el énfasis en la creatividad y en las cualidades personales del terapeuta. Otras ponen el acento más en la adherencia a una técnica determinada, como la cognitivo-conductual. Pero, jatención!, desde un extremo al otro, nadie dice que se puede practicar psicoterapia sin conocer a fondo los fundamentos teóricos y prácticos de la técnica que se practica.

Volvamos al problema de fondo. ¿Cuál psicoterapia para quién? ¿Estamos en condiciones de responder a esta pregunta con evidencia científica? En Estados Unidos, la división 12 de Psicología Clínica de la poderosa Asociación Psicológica Americana (APA) elaboró una lista de psicoterapias validadas empíricamente, esto es, psicoterapias cuyos procedimientos estaban bien definidos técnicamente y que disponían de estudios que demostraban su eficacia en trastornos también claramente especificados. Después tuvieron que dar un poco de marcha atrás y pasaron a hablar de psicoterapias sustentadas empiricamente (empirically supported), que indica un grado menor en las exigencias de validación. Los argumentos de la APA para llevar adelante este proyecto fueron: es de justicia difundir los resultados de las investigaciones, es lógico que la gente sepa qué tratamiento da qué resultado y es oportuno hacerlo ahora, porque existen los conocimientos necesarios. Agregan que esto va a beneficiar la atención de los pacientes y la planificación en salud, a mejorar la formación y a orientar la investigación.

Pero no hubo unanimidad, y ustedes pueden encontrar esta polémica en varios números de la Journal of the Society for Psychotherapy Research. En contra de esta lista se alzaron voces muy claras del lado de los terapeutas rogerianos y también de grupos psicoanalíticos, que sostenían que era prematura, inadecuada, que los estudios de eficacia basados en 
ensayos clínicos randomizados no podían aplicarse a terapias de larga duración en las que la motivación del paciente era fundamental. Proponían, en cambio, jerarquizar los estudios de efectividad, o sea, de la forma en la que los tratamientos funcionan en la práctica, en un contexto naturalista, ecológico. Sostenían que la cancha estaba flechada, muy sesgada y políticamente no era imparcial. Los criterios eran, por un lado, restrictivos, pero, a la vez, demasiado laxos. La polémica se centró en algunos puntos relacionados con las características de los ensayos clínicos controlados. Si bien las ventajas de este tipo de estudios para comparar distintos tratamientos no se discuten y están en la base de la medicina basada en pruebas, tampoco puede forzarse la realidad para que entre en los esquemas. Por ejemplo, en los ensayos controlados se busca tomar pacientes con el mismo trastorno. Pero los pacientes que buscan terapia dinámica o no directiva (rogeriana) muchas veces vienen más con un problema que con un trastorno diagnosticable; o existe un grado de comorbilidad importante, etcétera. Por otra parte, esas terapias jerarquizan la peculiaridad de cada caso, lo que hace difícil la sistematización diagnóstica. Volviendo al ejemplo de la depresión, ¿debe ser igual el tratamiento de una depresión caracterológica, ligada a conflictos intra e interpersonales crónicos, que una depresión mayor aguda en la que la asociación libre del paciente está seriamente inhibida? Es claro que en el primer caso parece más adecuado un tratamiento de orientación psicoanalítica, mientras que en el segundo se requiere una intervención más activa del terapeuta que la que se da en los análisis clásicos.

Otro punto álgido tiene que ver con la estandarización o manualización de los procedimientos. Mientras terapias como la cognitivoconductual se prestan con más facilidad a la manualización, otras como la rogeriana o la psicoanalítica dejan más lugar a la creatividad del terapeuta. Entonces, lo que para unos es una virtud para otros es una limitación. Notemos que no se discute que haya que establecer cuáles 
son las psicoterapias efectivas y cuál es la evidencia que las sostiene. Lo que se discute es cómo hacerlo. Leyendo las distintas posiciones, uno tiene la impresión de que, si bien hay discrepancias esenciales, también hay ciertos acuerdos fuertes en el punto medio, que pueden servir para seguir adelante. Por ejemplo, si bien hay que tomar en cuenta la variabilidad individual, también es cierto que existen categorías diagnósticas que agrupan a los individuos. Si bien existe la creatividad del terapeuta con cada paciente concreto, también es cierto que hay ciertos criterios generales y cada técnica recomienda cierta forma de intervenir y desaconseja otras, etcétera.

¿Adónde conducen estos consensos? El acuerdo más general es que hace falta más investigación, con nuevas y más potentes metodologías y con diseños más amplios que permitan tomar más en cuenta las características reales de los tratamientos. Por el momento, existen ciertos protocolos o guías para la práctica que reúnen en forma de recomendaciones los criterios más firmes en este campo. Ellos toman muy en cuenta la motivación del paciente. Establecen, por ejemplo, que si la persona busca resolver puntualmente los síntomas que lo limitan (ataques de pánico, fobias, depresión, etcétera) sin entrar a revisar su historia y sus conflictos, lo más adecuado es una terapia cognitivo-conductual. Si, en cambio, la persona desea comprender mejor su historia y sus dificultades intra o interpersonales y sus conflictos, entonces es recomendable que se derive hacia las terapias de orientación psicoanalítica. Y, por último, es necesario decir que existen formas de complementación entre estos distintos procedimientos e incluso la búsqueda de formas de integración, como la terapia cognitivo-analítica de Ryle.

En definitiva, creo que para el psicoanálisis se plantea un doble desafío. Por un lado, está el desafío de sustentar y decir con más precisión los resultados terapéuticos a corto y largo plazo. Afortunadamente, están en marcha proyectos de investigación sistemática que permitirán disponer 
de evidencia más segura que la sola impresión clínica de los analistas individuales (Fonagy, 1998; Galatzer-Levy et al., 2000).

El segundo desafío es el de admitir que existen otros recursos terapéuticos que son eficaces en ciertos casos y que, en consecuencia, es necesario elaborar una teoría general del cambio psíquico. Y que esta, tomando en cuenta lo que el psicoanálisis permitió descubrir sobre las procesos que conducen al enfermar y al curar, y tomando en cuenta el dolor psíquico, la sexualidad, la agresividad y los procesos del desarrollo, lleve a construir una teoría más general del cambio psíquico que permita dar cuenta no solo de lo que hace el psicoanálisis, sino también de lo que se puede lograr con otros recursos del campo de la salud mental, y lo que aportan la vida y los procesos espontáneos relacionados con la resiliencia y la capacidad de recuperación y de avance.

¿Cómo se vincula todo esto a nuestro medio? Creo que los desarrollos que hemos visto nos obligan a varias cosas. En primer lugar, a establecer con más claridad cuáles son las técnicas psicoterapéuticas que existen en nuestro medio, qué tipo de evidencia las avala y cuáles son los requisitos de formación necesarios para practicarlas. Esto no solo nos lo debemos entre nosotros: se lo debemos principalmente a los pacientes. Es por esa razón que esta discusión no debe ser solo una discusión interna o quedar limitada a grupos con intereses corporativos en el tema. Debe incluir a las autoridades sanitarias y educativas y garantizar la defensa de los usuarios.

En segundo lugar, creo que nos debemos estudios sistemáticos de los resultados de las distintas psicoterapias en nuestro medio. No alcanza con la evidencia que proviene de otros estudios. Debemos estudiar cómo funcionan en nuestro medio. Por último, hace falta que estudiemos a nivel clínico el mecanismo del cambio psíquico en las distintas psicoterapias. En realidad, muchos de los puntos que habría que discutir en cuanto al cómo del cambio entre las distintas psicoterapias los encontramos 
también como interrogantes dentro del psicoanálisis. Por ejemplo, acostumbramos decir que el psicoanálisis actúa per via di levare, esto es, levantando represiones, y no per via di porre, esto es, agregando la influencia del terapeuta. Pero es bueno preguntarnos cuánto de esto es realmente así y cómo se combina la acción de la interpretación con las distintas formas del apoyo (sugestión, educación, ejemplo, etcétera) y con lo que aporta la relación terapéutica misma. ¿Cuándo estos distintos factores de cambio contribuyen a que el paciente desarrolle su self verdadero? ¿De qué manera? Creo que, aun en forma hipotética, es apasionante comparar distintas psicoterapias desde este punto de vista, como lo hace Fonagy (1998), y tomar en cuenta la experiencia en nuestro medio.

A lo largo de esta charla he dejado tal vez demasiadas cosas en el tintero, pero no es posible hacer otra cosa en una exposición oral.

\section{ADENDA (FEBRERO DE 2021)}

Al releer esta exposición, realizada hace veinte años, me sorprendió comprobar la vigencia de las preguntas planteadas. Tal vez hoy habría incluido nuevos problemas u otros autores de referencia. Por ejemplo, hubiese mencionado investigaciones como las de Shedler y las de Leichsenring, que confirman el valor de las psicoterapias dinámicas. Abordaría la cuestión de los factores comunes entre distintos enfoques terapéuticos. A la vez destacaría la necesidad de guías para la práctica clínica en psicoterapia (Bernardi et al., 2004), que destaquen aquellas psicoterapias que Lambert y otros llamaron de bona fide, o sea, que poseen base teórica, formación acreditada y estudios de resultados, y que señalan la necesidad de distinguirlas de las que no ofrecen esas garantías. Pero estos y otros posibles agregados no alteran lo esencial del planteo ni la vigencia de las preguntas formuladas. De hecho, durante el tiempo transcurrido, 
he continuado reflexionando en torno a estas preguntas. No puedo, por cierto, decir que haya logrado resolverlas, pero sí debo afirmar que ellas me han llevado a explorar nuevos caminos durante este tiempo. De estos caminos quisiera hablar en esta adenda.

La exposición de 2002 parte de la pregunta de qué es lo que nos permite afirmar que nuestro trabajo sirve al paciente y para qué tipo de paciente es útil determinado tratamiento. Esto trae a su vez la pregunta de cómo describir lo que le pasa al paciente.

Junto con Berta Varela, Delfina Miller, Rosa Zytner, Laura de Souza y Rosario Oyenard hemos desarrollado el concepto de Formulación Psicodinámica del Caso (Bernardi et al., 2016). Esta noción es necesaria pues los diagnósticos tradicionales tipo DSM O CIE no logran transmitir los aspectos personales del paciente. De hecho, el DSM-5 advierte explícitamente que sus diagnósticos deben ser colocados en el marco de una formulación clínica del caso. Sin embargo, esta noción no es, o no era, frecuentemente utilizada en nuestro medio, lo que nos llevó a desarrollarla. La Formulación Psicodinámica del Caso intenta, desde una perspectiva psicoanalítica, dar en forma breve y conceptual una respuesta a las preguntas que plantea el tratamiento de todo paciente: qué le pasa al paciente, a qué se debe (en el sentido de cuáles son los factores predisponentes, precipitantes, perpetuantes o de protección), cómo pensamos tratarlo y qué resultados es posible comprobar.

Cada una de estas preguntas encierra asimismo numerosas cuestiones tanto a nivel teórico como práctico. Por ejemplo, exige analizar los conceptos utilizados, comparando las categorías usadas por los principales sistemas diagnósticos psicoanalíticos actuales (OPD-2 y PDM-2) y la versión alternativa (parte III) del DSM-5 y el nuevo modelo de los trastornos de la personalidad que aporta el CIE-11 (Bernardi et al., 2016). Esto, a su vez, abre nuevas cuestiones tanto teóricas como prácticas, lo que ha llevado a algunos de los autores a explorar la posibilidad de publicar un nuevo volumen, 
en el que se examinen nuevos aspectos conceptuales, así como los resultados prácticos que ha tenido en nuestro país el uso de la formulación del caso en la práctica privada e institucional.

La pregunta acerca de cómo evaluar el progreso de un tratamiento analítico se volvió central en mi trabajo en el Comité de Observación Clínica y en el Comité de Investigación de la Asociación Psicoanalítica Internacional (IPA). A propósito, planteé una guía para la discusión grupal de los efectos de un análisis tomando en cuenta material de distintos períodos. La he denominado Modelo de los Tres Niveles para la observación de las transformaciones del paciente, abreviado 3-LM (Bernardi, 2015). Este modelo fue adoptado por el Comité de la IPA y está siendo aplicado en diversos países, lo que ha dado lugar a nuevos desarrollos y publicaciones (Fitzpatrick Hanly et al., en prensa). Los tres niveles de la discusión grupal parten de la experiencia clínica y continuamente vuelven a ella para comprobar las hipótesis manejadas.

En el primer nivel, fenomenológico, importa la escucha analítica tal como se da en el analista y en el grupo de discusión. En el segundo nivel se busca una conceptualización de las dimensiones en las que ocurre el cambio del paciente (o su no cambio o empeoramiento). Las categorías diagnósticas psicoanalíticas que utiliza el 3-LM en este segundo nivel son muy similares a las que propone la Formulación Psicodinámica del Caso y están tomadas de las mismas fuentes. En el tercer paso o nivel la discusión grupal busca clarificar los focos del tratamiento y los posibles mecanismos de cambio, cotejando hipótesis teóricas sugeridas por diferentes enfoques teóricos y por las teorías implícitas y los modelos de trabajo personales del terapeuta. En diferentes países, al igual que lo que ocurrió en experiencias realizadas en AUDEPP y APU, es usual que las preguntas que propone el 3-LM lleven a que los grupos discutan durante varias horas el material clínico, con una actitud de investigación que busca descubrir nuevas perspectivas y comprobar hasta dónde encuentran apoyo en el material clínico. 
Esta actitud de indagación hace que tanto la Formulación Clínica del Caso como el Modelo de los Tres Niveles ayuden a construir puentes entre la investigación clínica y la investigación empírica sistemática (Bernardi, 2018). Este camino conduce a que la investigación clínica se vuelva más rigurosa y sistemática. El razonamiento clínico está orientado por hipótesis teóricas, pero resulta irremisiblemente sesgado si estas hipótesis se toman como verdades incuestionables y se busca que siempre queden confirmadas por el material clínico. El movimiento deductivo, de arriba hacia abajo, desde la teoría a la clínica, debe complementarse con una búsqueda inductiva que parta de la experiencia misma tal como ella se presenta en la práctica clínica. Este movimiento de abajo hacia arriba es el que tiene la última palabra y debe poner a prueba las hipótesis de las que se partió. Tienen especial importancia los procesos abductivos, que nos permiten encontrar la mejor explicación para determinado problema clínico singular. Cuando la investigación clínica se realiza en forma rigurosa y sistemática, resulta más fácil establecer puentes con otras formas de investigación. Andrea Rodríguez Quiroga y otros autores han utilizado el 3-Lm para la Investigación de Caso Único, en la cual el tratamiento de un determinado paciente es investigado a través de la triangulación de distintas metodologías, tanto cuantitativas como cualitativas; de esta forma, aumenta el nivel de la evidencia de las hipótesis en juego.

Evaluar el grado de evidencia de las hipótesis teóricas y clínicas es fundamental en investigación clínica. Para ello es necesario no solo examinar las características del material clínico, sino también sus posibles sesgos, omisiones y limitaciones (Bernardi y Pérez Suquilvide, en prensa). El grado o nivel de la evidencia es el que sustenta la fuerza de las recomendaciones que es posible realizar en base a la experiencia clínica. Estos problemas poseen una dimensión ética, porque para poder recomendar un tratamiento es necesario disponer de razones o comprobaciones que justifiquen que es el más adecuado. Volviendo a la pregunta 
«¿Qué psicoterapia?, ¿para quién?», debemos reconocer que nuestros conocimientos son aún muy limitados, pero a la vez tomar esto como un motivo para continuar en la búsqueda de nuevos caminos de avance.

\section{REFERENCIAS BIBLIOGRÁFICAS}

\section{¿Qué psicoterapia?, ¿para quién?}

\section{Algunos avances en un largo camino}

DeRubeis, R. J., Gelfand, L. A., Tang, T. Z. y Simons, A. D. (1999). Medications

Versus Cognitive Behaviour Therapy for Severely Depressed Outpatients: Mega-Analysis of Four Randomized Comparisons. The American Journal of Psychiatry 156(7), 1007-1013. https://ajp. psychiatryonline.org/doi/full/10.1176/ajp.156.7.1007

Elkin, I., Shea, M. T., Watkins, J. T., Imber, S. D., Sotsky, S. M., Collins, J. F., Glass, D. R., Pilkonis, P. A., Leber, W. R., Docherty, J. P., Fiester, S. J. y Parloff, M. B. (1989). National Institute of Mental Health Treatment of Depression. Collaborative Research Program. General Effectiveness of Treatments. Archives of General Psychiatry, 46, 971-982. https://jamanetwork.com/journals/jamapsychiatry/ article-abstract/494730

Fonagy, P. (ed.) (1998). An open door review on psychoanalytic outcome studies. Report prepared by the Research Committee of the IPA al the request of the President. IPA. www.researchgate.net/publication/51999000_ An_open_door_review_of_outcome_studies_in_psychoanalysis

Galatzer-Levy, R., Bachrach, H., Skolnikoff, A. y Waldron, S. (2000). Does Psychoanalysis Work? Yale University Press. 
Roth, A. y Fonagy, P. (1996). What works for whom? A Critical Review of Psychotherapy Research. The Guilford Press.

Sperry, L., Brice, P. L., Howard, K. I. y Grisson, G. R. (1996). Treatment Outcomes in Psychotherapy and Psychiatric Interventions (Mental Health Practice Under Managed Care, volume 6). Brunner/Mazel.

\section{Adenda (febrero de 2021)}

BERNARDI, R. (2010). DSM-5, OPD-2 y PDM: Convergencias y divergencias entre los nuevos sistemas diagnósticos psiquiátricos y psicoanalíticos. Revista de Psiquiatría del Uruguay, 74(2), 179-205. www.spu.org.uy/ revista/dic2010/10_bernardi.pdf

Bernardi, R. (2015). El Modelo de los Tres Niveles (3-Lm) para observar y describir las transformaciones del paciente. En Tiempo de cambio. Indagando las transformaciones en psicoanálisis: el modelo de los tres niveles. Karnak.

Bernardi, R. (2018). ¿Qué es la investigación clínica? En Psicoanálisis latinoamericano contemporáneo. APA.

Bernardi, R., Defey, D., Garbarino, A., Tutté, J. y Villalba, L. (2004). Guía Clínica para la Psicoterapia. Revista de Psiquiatría del Uruguay, 68(2), 99-146. www.spu.org.uy/revista/dic2004/02_guia.pdf Bernardi, R., Varela, B., Miller, D., Zytner, R., De Souza, L. y Oyenard, R. (2016). La formulación psicodinámica de caso: su valor para la práctica clínica. Grupo Magro.

Bernardi, R. y Pérez Suquilvide, L. (en prensa). Assessing Strengths and Limitations of Clinical Evidence in a Psychoanalytic Clinical Material. En M. A. Fitzpatrick Hanly, M. Altmann y R. Bernardi (eds.), Change over time and how change can be observed in psychoanalysis. Karnak.

FitzPatrick Hanly, M. A., Altmann de Litvan, M. y Bernardi, R. (eds.) (en prensa). Change through time in Psychoanalysis. Karnak. 
